\title{
CRISPR/Cas9 gene editing technology and its application to the coronavirus disease (COVID-19),
} a review

\author{
Saeid CHEKANI-AZAR ${ }^{1}$, Ehsan GHARIB MOMBENI ${ }^{2}$, Mastewal BIRHAN ${ }^{3 \star 凶}$ and Mahshad YOUSEFI ${ }^{4}$ \\ ${ }^{1} \mathrm{PhD}$, Faculty of Veterinary Medicine, Animal Physiology, Atatürk University, Turkey \\ ${ }^{2} \mathrm{PhD}$, Department of Pathobiology, Shahid Chamran University of Ahvaz, Iran \\ ${ }^{3} \mathrm{PhD}$, College of Veterinary Medicine and Animal Science, Department of Veterinary Paraclinical Studies, University of Gondar, Ethiopia \\ ${ }^{4} \mathrm{MD}$, Hamadan University of Medical Sciences, Hamadan, Iran \\ Corresponding author's Email: maste675@gmail.com; (1) ORCiD: 0000-0002-0984-5582
}

\begin{abstract}
Introduction. Clustered-Regularly Interspaced Short Palindromic Repeats (CRISPR), and CRISPR associated (Cas) protein (CRISPR/Cas) structures were first identified in E. coli in 1987 and guard prokaryotic cells from any invading pathogens, harmful events and plasmids by recognizing and cutting foreign nucleic acid sequences that contain short palindromic repeats spacer sequences. Several genome editing approaches have been developed based on these mechanisms; the most recent is known as CRISPR/Cas. Before the CRISPR technique was revealed in 2012, editing the genomes of plants and animals took many years and cost hundreds of thousands of dollars. Thus, CRISPR/Cas has attracted significant interest in the scientific community, especially for disease diagnosis and treatment, as it is quicker, less expensive and more precise than other genome editing approaches. The evidence from gene mutations in specific patients generated using CRISPR/Cas can assist in the prediction of the optimal treatment schedule for individual patients and for innovation purposes in other researches like replication in cell culture of coronaviruses like severe acute respiratory syndrome coronavirus-2 (SARS-CoV2 or COVID-19). However, in numerous situations, the effects of the furthermost significant driver mutations are not yet understood and interpretation of the optimal treatment is impossible. CRISPR/Cas classifications feature highly sensitive and selective tools for the detection of various target genes. When we see the next steps of genomic research, it is obvious that genome-wide association studies are relatively new way to identify the genes involved in human disease. Furthermore, CRISPR/Cas provides a tool to manipulate non-coding regions and will thus accelerate examination of these poorly characterized regions of the genome and play a vital role in the progress of whole genome libraries. Aim. We aimed to review the history of CRISPR/Cas, the mechanisms of CRISPR techniques, its current status as a tool for studying both natural mutations and genomic manipulations, and explore how CRISPR/Cas may improve the treatment of diseases.
\end{abstract}

\section{Review Article \\ PII: S225199392000001-10 \\ Rec. 22 December 2019 \\ Rev. 15 January 2020 \\ Pub. 25 January 2020}

\section{Keywords}

\section{CRISPR/Cas9,}

DNA-targeting,

Palindromic,

Plasmids,

Genome sequencing,

SARS-CoV2,

COVID-19

\section{INTRODUCTION}

Clustered regularly interspaced short palindromic repeats (CRISPR) were first discovered in Escherichia coli in 1987 and later found in other bacteria species. The role of these repeat sequences remained unclear until, in 2005, several researchers described the similarities of the sequences ` DNA, leading to the hypothesis that the sequences are part of adaptive immune system in bacteria (CRISPR/Cas9 for cancer research and therapy).

As you know, CRISPR is also refers to an adaptive immune response in bacteria and archaea that is cast-off to target and cut down viral DNA by using endonuclease in specific ways. By reengineering this immune response to target parts of genetic material, scientists could make extremely precise genetic alterations tailored to the type of cell. This is the basis of CRISPR therapeutic and diagnostic platforms [1].

CRISPR and CRISPR-associated proteins characterize the immune system of archaea and bacteria, and deliver protection against invasive nucleic acids, DNA, or RNA from phages, plasmids, and other exogenous DNA elements. At this time, two different classes, six types, and 21 subtypes of CRISPR-Cas systems have been identified [2]. The length of the repeat sequences varies between 25 and $40 \mathrm{nt}$, whereas the length of the spacer sequences varies between 21 and $71 \mathrm{nt}[3]$. 
For the success of these, innumerable methods have been fashioned for targeted gene editing in cell and animal models. These include Zinc-Finger Nucleases (ZFNs), Transcription Activator-Like Effector Nucleases (TALENs). But, Clustered-Regularly Interspaced Short Palindromic Repeats (CRISPR), and CRISPR Associated (Cas) protein (CRISPR/Cas) is a rapid, simple, and often extremely efficient gene editing method [4]. That is why it is a powerful method for making changes and simple to manipulate the genome of many organisms [5]. Form these scientific truth, we can understand the advantage of genome editing tools made possible to modify a genomic DNA in a targeted fashion by applying of CRISPR/Cas [6].

Recent developments in CRISPR/Cas gene editing have made it possible to introduce precise changes into a wide variety of genomes, including those of dsDNA viruses; in addition to this, it is pursuing relies on the coexpression of a prokaryotic Cas nuclease and an associated guide RNA (gRNA) sequence. Because, once gRNAtargeted Cas, it creates a double-stranded break (DSB) in the genome, by making of two main repair processes compete to repair the damage, leading to with some modifications of from the original sequence [7].

The study of gene function in the recent decades has trusted on the generation of systemic and conditional gene knockouts to disrupt gene expression especially in coronaviruses. For example, de Wilde et al. [8] investigated the cyclophilin A (СурA) protein-dependence in the replication of three related nidoviruses in the same cell line (Huh7), in which CypA expression was knocked-out using CRISPR/Cas9 gene editing technology (Huh7-СypAKO cells). Therefore, even if successful studies and some innovation ideas and solution were done, but, it has been challenging to knockout a gene in specific cell types in the brain. The final target and goals of CRISPR was to revolutionize the treatment of hereditary diseases based therapeutics. Finally, the aim of this systematic review paper is to summarize the application of CRISPR/Cas and its overall immune signal transduction and also discussing gene editing technology against novel diseases like coronavirus (COVID-19).

\section{Current status and significance of CRISPR/Cas and immune cells}

\section{Types of CRISPR/Cas systems}

CRISPR/Cas systems have been categorized in details such as class 1 which utilizes multi-protein effector complexes and class 2 which utilizes single-protein effectors. Class 1 is further divided into different types I, III, and IV, while class 2 includes types II, V, and VI. It can also be divided into 19 different subtypes and it is likely to continue to expand as new CRISPR/Cas systems are identified. The greatest corporate Cas protein used for functional broadcast is a type II single-protein effector derived from Streptococcus pyogenes (SpCas9). It is a type of guide RNA to succor in successfully cleaving the target gene [9].

This choice stems from the fact that types I and III are composed of a number of Cas proteins, although the Cas9 protein is the only protein constituent of type II CRISPR/Cas systems [10]. One of the difficulties in classifying and applying for the success is designing and construction of novel CRISPR/Cas systems with the researcher effort to demarcate targeting sequences in a simplified manner [11]. Type II system is composed of trans-activating crRNA (tracrRNA). CRISPR loci are prepared of "repeat-spacer" array and adjacent to small clusters of Cas genes including Cas9 endonuclease [12]. From this perspective, Cas proteins are of great necessity for the functioning of CRISPR/Cas immune system and are considered as indicators of the system's activity [13].

\section{CRISPR in human pluripotent stem cells}

Human civilization has always faced two basic challenges: That are disease and hunger. Efforts to prevent or treat disease and accomplish food security endure. Despite of the progress made food insecurity and many. Diseases and pests persist; these problems have proved difficult to solve using available technologies. Nevertheless, hopes are high for solutions to many of these challenges with the advent of CRISPR technology [14]. There are different human cells those are, immortalized MRC5-hTERT fibroblasts were cultured in Dulbecco's modified Eagle medium, supplemented with fetal bovine serum (10\%), 1\% penicillin-streptomycin and $4.5 \mathrm{~g} / \mathrm{L}$ glucose, [7].

\section{Overview of CRISP-Cas-mediated genome editing}

Engineered nucleases, such as ZFN, TALEN, and Cas9, can induce DNA double-strand breaks (DSBs) at specific genomic loci, which are subsequently repaired by one of at least two endogenous cellular DNA repair pathways: non-homologous end joining (NHEJ) and homology-directed repair (HDR) [15]. Over the past two decades, the discovery of programmable DNA binding proteins (ZFs, Zinc fingers, and transcription activator- 
like effectors [TALEs]) has paved the way for the development of genome editing tools. Compared with ZFs, which contain repeated Cys2-His2 ( $2 \mathrm{H} 2)$, DNA binding domain (DBD) adapted from a family of eukaryotic transcription factors (TFs) [16].

The CRISPR/Cas9 genome-editing technology is derived from the adaptive immune response in prokaryotes. This method has several advantages over conventional genome-editing technologies, including: a) simplicity in target designing, b) regulation, and c) the ability to target multiple genes. CRISPR consists of two major components: sgRNA and Cas protein. The sgRNA consists of a sequence, also called as scaffold. sgRNA includes a Cas enzyme binding site and a spacer sequence, called the target sequence, which is specific to the gene of interest. The spacer sequence contains $\sim 20$ nucleotides and is followed by protospacer adjacent motif (PAM), a 2-6-base pair DNA sequence immediately following the DNA sequence targeted by the Cas9 nuclease in the CRISPR. PAM sequences vary based upon the type of Cas9 enzyme [17].

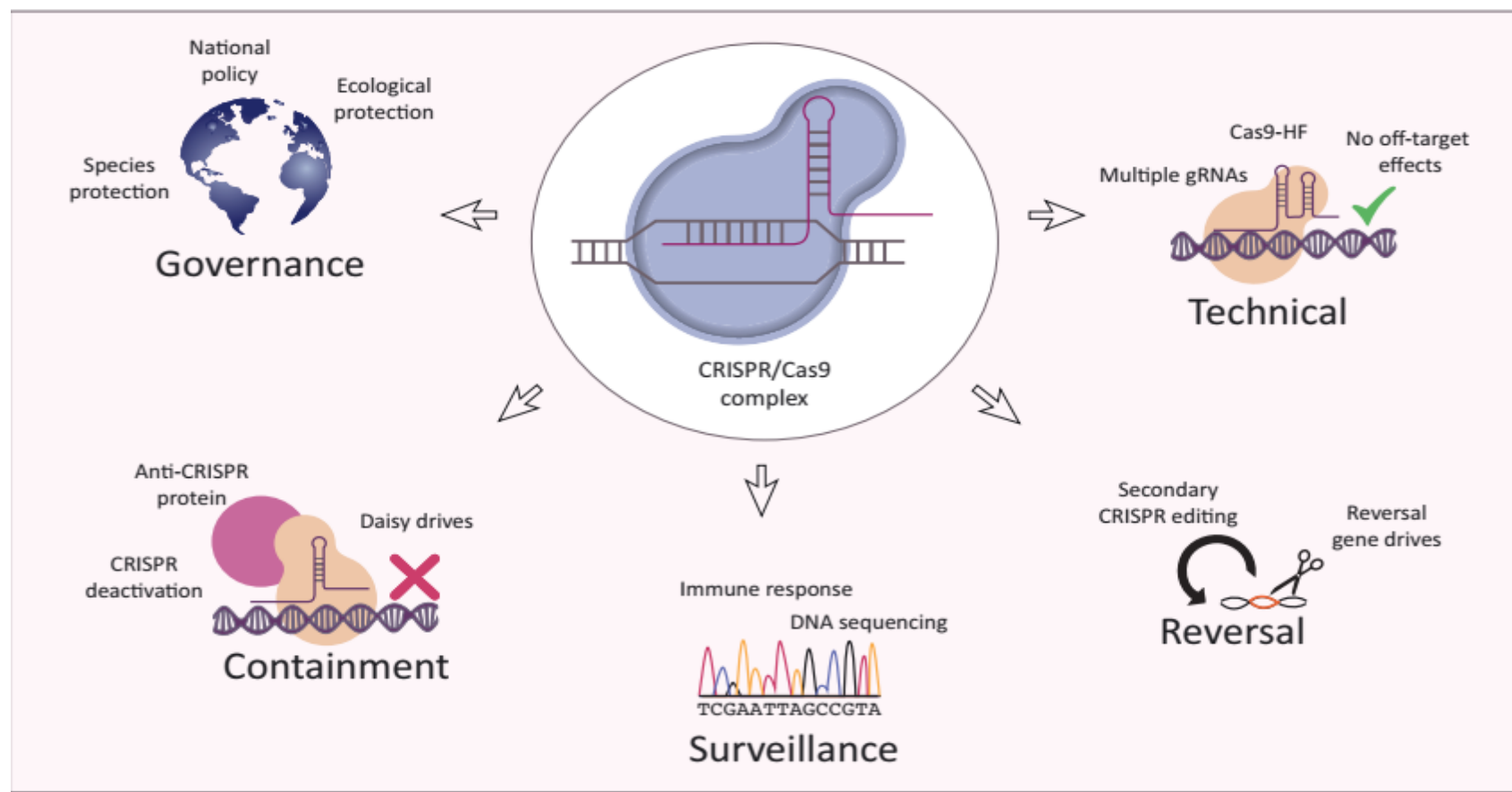

Figure 1. Promoting safety in genome editing [18].

\section{Editing efficiency}

There are a number of changes and factors that contribute for the difficulty of genome editing. The second major challenge in the genome editing is efficiency. The therapeutic efficacy of genome editing will be enhanced by improving the editing rates. For CRISPR/Cas, the activity of double-stranded break (DSB) restoration pathways plays an important regulatory role in its editing rates.

Due to the different endogenous cellular DNA repair mechanisms mediated by non-homologous endjoining (NHEJ) and homology-directed repair (HDR), there are definitely more editing efficiency differences in both different cell types and also cellular states. HDR is differently operates during the S/G2 phase while NHEJ is active during the whole cell cycle, thus, it is generally more efficient for generating indels to knockout carcinogenic genes than HDR. The efficiency of HDR, as precise gene adjustment, is relatively lethargic depending on both homology arm length and DNA template type. HDR templates are typically single-stranded oligonucleotides or plasmids containing alleles and delivery of HDR templates has been accomplished with viral or non-viral vectors [19].

\section{CRISPR/Cas9 toolsets}

There are a number of online CRISPR tools and resource available, provided by both academic and commercial institutions, with a list and its application of the most widely used resources provided in Table 1. The function of these CRISPR resources is primarily to identify and recognized gRNAs, and provide the essential information required to select gRNAs for experimental applications. While some of the tools may be able to "mention" gRNAs for specific purposes. However for most applications, these CRISPR tools are just one component of the overall design process that starts with the identification of target locations [20]. 
Table 1. List of common CRISPR resources [20].

\begin{tabular}{ll}
\hline Name & URL \\
Addgene & http://www.addgene.org/crispr/ \\
\hline Benchling & https://benchling.com/crispr \\
\hline BreakingCas & http://bioinfogp.cnb.csic.es/tools/breakingcas/ \\
\hline Broad Institute GPP & https://portals.broadinstitute.org/gpp/public/ \\
\hline CHOPCHOP & http://chopchop.cbu.uib.no/ \\
\hline CRISPOR & http://crispor.net/ \\
\hline CrisFlash & https://github.com/crisflash/crisflash \\
\hline Deskgen & https://www.deskgen.com \\
\hline E-CRISP & http://www.e-crisp.org/E-CRISP/ \\
\hline Horizon Discovery & https://dharmacon.horizondiscovery.com/gene-editing/crispr casg/crispr-design-tool/ \\
\hline IDT & https://sg.idtdna.com/site/order/designtool/index/CRISPR_CUSTOM \\
\hline Microsoft Research CRISPR & https://crispr.ml \\
\hline RGEN Tools & http://www.rgenome.net/ \\
\hline Synthego & http://design.synthego.com \\
\hline WTSI Genome Editing (WGE) & https://www.sanger.ac.uk/htgt/wge/ \\
\hline WTSI: Wellcome Trust Sanger Institute; RGEN: RNA-Guided Endonucleases; GPP: Green Public Procurement
\end{tabular}

\section{CRISPR Applications and Design}

For the applications systems of all CRISPR/Cas is depend on the gRNA's ability to recognize specific target sequence, even though not all applications are dependent on the cleavage activity of the Cas-endonuclease. Because of both knock-in (KI) and knock-out (KO) solicitations are related to the formation of a DSB to insert DNA or either delete $[20,21]$. Designing needs about the basic principles of CRISPR that involved to identifying a suitable target region or regions, tracked by the choices of suitable gRNAs based on positioning the target region: off-target and on-target scoring. This is very important for the applies of both targeted design and large-scale design approaches, even though the methods used for selecting and targeting gRNAs can be exact different between these approaches [20].

There are invaluable information for systematic analyses of the genes which can be achieved by parameters like molecular characterization, mechanics behind biological processes, and screening of therapies and responses to drugs [22]. When we see for example like cancer: it is a genetic disease stemming from cumulative genetic/epigenetic aberrations, it is rational for us to envisage that modifying the oncogenic genome abnormalities through CRISPR/Cas might characterize a promising therapeutic strategy against cancer [19]. Introducing only a single DSB to stimulate the DNA-repair mechanisms can be sufficient, but even introducing more than one DSB for large deletions or insertions may be necessary. In the lack of a repair template, NHEJ pathway may introduce small idles within a coding sequence, which can disrupts protein translation by a frame shift and thus knockout a gene. An ideas suitable for DNA repair template is on condition that, the same double-stranded break (DSB) may be repaired by homology-directed repair (HDR), leading to the insertion or knock-in of any exogenous DNA included within the template. In contrast, CRISPR repression and activation (CRISPRa/i) uses a catalytically dead or inactivated Cas-endonuclease to target transcriptional repressor complexes or activator to a specific site within the promoter region [20].

Today, the application of the CRISPR/Cas9 method has expanded into a variety of areas, including agriculture, husbandry, disease patterns and targeted therapies. This section emphasizes the therapeutic aspects for diseases with a genetic basis, especially monogenic disorders. In the ex vivo approach, the targeted cell population is removed from the patient's body, and the designed nucleases are used to induce the desired changes and then returned to the patient by grafting. Avoiding issues such as transplant rejection and immune responses are important benefits of this method. Using in vivo gene therapy, the genomic modifying factors, such as programmatic nucleases and patterns, are put directly into the patient [23].

In the case of Cas9; it is used to change endogenous genes which previously were problematic to be genetically manipulated, resulting in a more efficient and faster production of transgenic models. Those transgenic models that can be produced simply by injecting fertilized zygotes with customized Cas9 and transcribed single guide RNA for the manipulation of alleles and accomplish desired alterations in mammalians. Casg is also used in cellular models to study for the treatment and diagnosis of polygenic diseases such as diabetes and autism due to multiplexing properties [24]. 


\section{CRISPR/Cas9 gene editing technology applications against diseases like COVID-19}

CRISPR/Cas biotechnology is now expansively and broadly applied across numerous disciplines, including basic sciences, food/crop development, fuel generation, drug development, human genome engineering and gene editing technology against novel diseases like coronavirus (COVID-19 or SARS-CoV2) [8, 14, 19, 22, 25, 26].

The Nidovirales that are consisted of the ronivirus, mesonivirus, arterivirus, and coronavirus families have minor or major economic and societal effects. In 2002-2003, the highly pathogenic outbreaks (ongoing), human coronaviruses (HCoVs), severe acute respiratory syndrome coronavirus (SARS-CoV) and Middle East respiratory syndrome coronavirus (MERS-CoV) occurred in zoonotic and transmitted to the human population, with alarming mortality. SARS-CoV outbreaks led to more than 8000 confirmed cases within a few months, (mortality rate: $~ 10 \%$ ) [27] and for the MERS-CoV resulted in over 2000 human cases ( 35\% mortality rate) (http://www.who.int/emergencies/mers-cov/en/) [28]. But in December 2019, another pathogenic HCoV, 2019 named novel coronavirus (2019-nCoV, COVID-19 or SARS-CoV2), was recognized in Wuhan, China [29, 30], and has caused serious mortality rate of $\sim 35 \%$ (https://www.worldometers.info/coronavirus/). In most patients, COVID-19 infection is associated with a cytokine storm [27, 28, 30-33]. In recovered cases, the excessive immune responses lead to long-term lung damage and fibrosis, causing functional disability and reduced quality of life $[34,35]$. There is no specific anti-viral treatment, and designing effective antiviral drugs against COVID-19 will take several years to develop and evaluate and also a range of existing host-directed safe therapies [36-38] could potentially be repurposed to treat COVID-19 infection. Hence, an urgent funding and scientific investments into advancing novel therapeutic interventions for coronavirus infections, is required.

Therefore, the lack of proper strategies to control nidovirus-induced disease, increase the importance of knowledge of these viruses' replications and also their interactions with the host cell and specially role of CRISPR-Cas gene editing technology against these viruses. Nidoviruses are +RNA viruses with different genomes (arteriviruses with 13 to $16 \mathrm{~kb}$, and coronaviruses with 26-34 kb) [39, 40], with a complex genome translation for producing the nsps (polyprotein precursors of the nonstructural proteins) as well as a nested set of subgenomic (sg) mRNAs to express the structural proteins [41, 42]. Nidoviral nsps, probably with various host factors, assemble into replication and transcription complexes (RTCs) that drive viral RNA synthesis [43-46]. These RTCs are probably associated with a virus-induced network of endoplasmic reticulum-derived membrane (ERDM) structures, including many double-membrane vesicles [43, 47-49].

Nidovirus replication depends on membrane trafficking, cellular proteins and membranes, and host signaling pathways or host cell factors and processes [50-52]. The cyclophilin (Cyp) protein family members have been implicated in nidovirus replication. The Cyps family of peptidyl-prolyl isomerases (PPIases), act as chaperones to facilitate protein folding, trafficking and immune cell activation [53, 54]. Cyps family that are ubiquitous and abundant cytosolic proteins, are especially expressed CypA, an important factor that implicated in the replication of various RNA viruses. CypA have also specific roles in various viruses like human hepatitis $C$ virus (HCV) and immunodeficiency virus-1 (HIV-1) infections. It assists HCV polyprotein processing, interacts with HCV NS5A for remodelling of cellular membranes into HCV replication organelles, and stabilizes HIV-1 capsids to promote nuclear import of the HIV-1 genome [55].

Studies with general Cyp inhibitors such as cyclosporine A (CsA) showed that, cyclophilins were initially implicated as host factors in nidovirus replication. In cell culture, the replication of a variety of arteriviruses and coronaviruses was found to be strongly inhibited by lowmicromolar concentrations of CsA and the nonimmunosuppressive CsA analogs Alisporivir (ALV) and NIM-811 [56-63]. And then nidovirus replication can depend specifically on CypA and/or CypB.

The replication in cell culture of the alphacoronaviruses feline coronavirus [64], the arterivirus equine arteritis virus (EAV) [65]; and human coronavirus (HCoV)-NL63 [56], and HCoV-229E [63] was reported to be affected by CypA knockout or knockdown. Finally, it seems that the normally cytosolic CypA was found to cosediment with membrane structures containing EAV RTCs, have a direct association with the arteriviral RNAsynthesizing machinery [65].

All the studies are depends on the nidoviruses types and cell lines, CypA expression levels, and readouts to measure viral replication efficiency. de Wilde et al. [8] investigated the CypA-dependence of the replication of three related nidoviruses in the same cell line (Huh7), in which CypA expression was knocked-out using CRISPR/Cas9 gene editing technology (Huh7-CypAKO cells). Using different cell lines, the replication of the arterivirus EAV [65] and the alphacoronavirus HCoV-229E [63], was previously concluded to depend on CypA. And the CypA dependence of betacoronaviruses like MERS-CoV has been documented for a first time with de Wilde et al. [8]. They reported that infection of Huh7-CypAKO cells with MERS-CoV revealed that its replication 
was only modestly affected by the absence of CypA, as opposed to equine arteritis virus which was strongly inhibited. While they stated that, HCoV-229E replication was not affected at all in the Huh7-CypAKO cells. They thus revealed major differences in CypA dependence of the arterivirus EAV in compared to other coronaviruses. There is a need to more evaluation of the CypA role in the replication of members of the latter virus family.

\section{The capacity of CRISPR diagnostics}

Diagnostic uses of CRISPR technologies help immense advantages in clinical effectiveness. A vast numbers of researchers have used CRISPR-based editing and diagnosis to correct the genetic basis of many diseases in isolated from the cells of animal models. The first groundswell of clinical trials using CRISPR enzymes to treat inherited disorders in humans involves eradicating a patient's cells, editing ex vivo, and rein fusing the corrected cells. Such ex vivo genome editing is currently the most technically feasible approach, and has the potential to treat devastating blood disorders like sickle cell disease and $\beta$-thalassemia. The ex vivo strategy also underlies cancer immunotherapies [66].

The role and function of CRISPR/Cas in gene editing in applications as diverse as fetal medicine, biodefense, and synthetic food makes for a stream of high-profile news. In 2018, several articles were published on the use of CRISPR in diagnostic tests for early-stage cancer detection or for infectious diseases. Further, it was seen as the most important innovation used to develop a rapid CRISPR-based assay for TB detection [25].

\section{CRISPR ethics}

Ethical decisions, particularly in biomedicine, are empirically informed and involve evaluating potential risk-benefit ratios, with the attempt of maximizing benefits while minimizing risk. To navigate ethical decision making, it is critical to consider the range of possible consequences, the probabilities of each instantiating, and the possible rationalizations driving results. The ethical concerns about CRISPR genome engineering technology are largely due to at least three important explanations. These include the possibilities of limited ontarget editing efficiency [67].

CRISPR/Casg is inexpensive, efficient and exact method to edit genes at the level of individual nucleotides, and also help to explore or explain many scientific questions. Moreover, this gene editing technology provides new potential treatments for many human diseases like novel coronavirus (COVID-19). In addition, the use of CRISPR/Cas9 gene editing technologies along with stem cells (i.e. induced pluripotent stem cells), can help to generate gametes for reproductive purposes or correct errors in their genome, and can also minimize the need for oocyte donation [68].

In 2017, the US National Academies of Sciences, Engineering, and Medicine Committee on Human Gene Editing published reviews of scientific, legal or ethical concerns about the amazing progress of gene engineering technology. The astonishing report, was that heritable genome editing help to modification of the germ line with the aim of generating a new human being who could therefore transfer the genomic change to future generations-hold be impermissible now but eventually could be justified for certain medical indications. Currently, create, destroy, or modification of human embryos to include heritable genetic changes for research purposes, is unlawful for U.S. federal funds. The NASEM is still implies that if safety risks guaranteed, clinical trials conceivable would commence [69].

\section{CONCLUSIONS AND RECOMMENDATIONS}

Form these systems review; we can appreciate the most important components of CRISPR RNAs (crRNAs) and Cas effector proteins. CRISPR will be a very important and crucial study area for disease diagnosis and treatment in the future, with the best potential for research in the scientific community. Beside this, it will be important for correcting the mutations and hereditary diseases concerning immune cells and their system disturbances; it will be important even for immunological tolerance to correct wrongly activated immune cells that fail to identify self and non-self, leading to cancerous disturbances of immune balance. It is very crucial in facilitating the etiology of diseases and checking their causative agents. From its adaption, it has revolutionized molecular biology and genetics in general. In combination with parallel developments of the necessary supportive techniques, modern biotechnology has led to useful genetic modifications of micro-organisms, plants and animals it has generated, inter alia, various new therapeutics and diagnostics. Owing to such advancements, we can study and work with this environment in an easier manner. CRISPR gene editing and correcting methods are used for this purpose. Based on these, we can forward some recommendations 
pertaining to future studies related to CRISPR-Cas: A) All researchers, academicians, international NGOs and funders, the scientific community as well as the government should focus on CRISPR/Cas-based diagnostics to enable this; B) In the future, we can also focus on pharmacogenomics. This will allow for the development of designer drugs to treat a wide range of health problems and disorders occurring as a result, including cardiovascular diseases, Alzheimer `s, HIV/AIDS, asthma, cancer and the worldwide pandemics like coronavirus (COVID-19).

\section{DECLARATIONS}

\section{Authors' contributions}

S.Chekani-Azar, E.Gharib Mombeni and M.Birhan* conceived the review, and M.Yousefi coordinated the overall activity and article processing.

\section{Conflict of interest}

The authors declare that there is no conflict of interest.

\section{Acknowledgment}

The authors' heartfelt thanks are given to University of Gondar, Research and Community Service V/President Office, Ethiopia; Collage of Veterinary Medicine and Animal Sciences, Atatürk University, Erzurum, Turkey; Department of Pathobiology, Shahid Chamran University of Ahvaz, Iran; and Hamadan University of Medical Sciences, Hamadan, Iran and finally Journal of Life Science and Biomedicine for the resource supporting and free of charge publication of the review article.

\section{REFERENCES}

1. Pan A and Kraschel KL. Kraschel. CRISPR diagnostics: Underappreciated uses in perinatology. Semin Perinatol. 2018; 42(8):525530. PMID: 30415764; DOI: https://doi.org/10.1053/j.semperi.2018.09.016

2. Hidalgo-Cantabrana C, Goh YJ and Barrangou R. Barrangou, Characterization and repurposing of type I and type II CRISPRCas systems in bacteria. J Mol Biol. 2018; 431(1): 21-33. DOI: https://doi.org/10.1016/j.jmb.2018.09.013; PMID: 30261168;

3. Qu D, Lu S, Wang P, Jiang M, Yi S, et al. Analysis of CRISPR/Cas system of Proteus and the factors affected the functional mechanism. Life Sci. 2019; 231: 116531. PMID: 31175856; DOI: https://doi.org/10.1016/j.lfs.2019.06.006

4. Mehravar M, Shirazi A, Nazari M and Banan M. Mosaicism in CRISPR/Cas9-mediated genome editing. Dev Biol. 2018; 445(2): 156-162. PMID: 30359560; DOI: https://doi.org/10.1016/j.ydbio.2018.10.008

5. Zhan T, Rindtorff N, Betge J, Ebert MP and Boutros M. CRISPR/Cas9 for cancer research and therapy. Semin Cancer Biol. 2019; 55(2019): 106-119. PMID: 29673923; DOI: https://doi.org/10.1016/j.semcancer.2018.04.001

6. Herai RH. Avoiding the off-target effects of CRISPR/cas9 system is still a challenging accomplishment for genetic transformation. Gene. 2019; 700: 176-178. PMID: 30898720; DOI: https://doi.org/10.1016/j.gene.2019.03.019

7. King MW and Munger J. Editing the human cytomegalovirus genome with the CRISPR/Cas9 system. Virology. 2019; 529: 186194. PMID: 30716580; PMCID: PMC6382551; DOI: https://doi.org/10.1016/j.virol.2019.01.021

8. de Wilde AH, Zevenhoven-Dobbe JC, Beugeling C, Chatterji U, de Jong D, et al. Coronaviruses and arteriviruses display striking differences in their cyclophilin a-dependence during replication in cell culture. Virology. 2018; 517: 148-156. PMID: 29249267; PMCID: PMC7112125; DOI: https://doi.org/10.1016/j.virol.2017.11.022

9. Ford K, McDonald D and Mali P. Functional genomics via CRISPR-Cas. J Mol Biol. 2019; 431(1): 48-65. PMID: 29959923; PMCID: PMC6309720; DOI: https://doi.org/10.1016/j.jmb.2018.06.034

10. Makarova KS, Haft DH, Barrangou R, Brouns SJ, Charpentier E, et al. Evolution and classification of the CRISPR-Cas systems. Nat Rev Microbiol. 2011; 9(6): 467. PMID: 21552286; PMCID: PMC3380444; DOI: https://doi.org/10.1038/nrmicro2577

11. Khatodia S, Bhatotia K, Passricha N, Khurana S and Tuteja N. The CRISPR/Cas genome-editing tool: application in improvement of crops. Front Plant Sci. 2016; 7: 506. PMID: 27148329; PMCID: PMC4835450; DOI: https://doi.org/10.3389/fpls.2016.00506

12. Yamaguchi Handde Lecea L. In vivo cell type-specific CRISPR gene editing for sleep research. J Neurosci Methods. 2018; 316: 99-102. DOI: https://doi.org/10.1016/j.jneumeth.2018.10.016. Epub 2018 Nov 12. PMID: 30439390; PMCID: PMC6380930;

13. Deveau $H$, Barrangou $R$, Garneau JE, Labonté J, Fremaux C, et al. Phage response to CRISPR-encoded resistance in Streptococcus thermophilus. J Bacteriol, 2008; 190(4): 1390-1400. PMID: 18065545; PMCID: PMC2238228; DOI: https://doi.org/10.1128/JB.01412-07

14. Ogaugwu CE, Agbo SO and Adekoya MA. CRISPR in sub-Saharan Africa: applications and education. Trends Biotechnol. 2019. 37(3): p. 234-237. PMID: 30100229; PMCID: PMC6378648; DOI: https://doi.org/10.1016/j.tibtech.2018.07.012

15. Nishiyama J. Genome editing in the mammalian brain using the CRISPR-Cas system. Neurosci Res. 2019; 141: p. 4-12. PMID: 30076877; DOI: https://doi.org/10.1016/j.neures.2018.07.003

16. Rahman S, Datta M, Kim J and Jan AT. CRISPR/Cas: An intriguing genomic editing tool with prospects in treating neurodegenerative diseases. Semin Cell Dev Biol. 2019. 96: 22-31. DOI: https://doi.org/10.1016/j.semcdb.2019.05.014; PMID: 31102655

17. Kaushik I, Ramachandran S and Srivastava SK. CRISPR-Cas9: A multifaceted therapeutic strategy for cancer treatment. Semin Cell Dev Biol. Elsevier. 2019; 96:4-12. DOI: https://doi.org/10.1016/j.semcdb.2019.04.018; PMID: 31054324; PMCID: PMC6829064 
18. Pineda M, Lear A, Collins JP and Kiani S. Safe CRISPR: Challenges and Possible Solutions. Trends Biotechnol. 2018; 37(4): 389401. PMID: 30352704; DOI: https://doi.org/10.1016/j.tibtech.2018.09.010

19. Chen M, Mao A, Xu M, Weng Q, Mao J, et al. CRISPR-Casg for cancer therapy: Opportunities and challenges. Cancer lett. 2019; 447: 48-55. PMID: 30684591; DOI: https://doi.org/10.3390/biomedicines6040105

20. Thomas M, Parry-Smith DandIyer V. Best practice for CRISPR design using current tools and resources. Methods, 2019; 164165(15 July): 3-17. PMID: 31152780; DOI: https://doi.org/10.1016/j.ymeth.2019.05.019

21. Fu BXH, Smith JD, Fuchs RT, Mabuchi M, Curcuru J, et al. Target-dependent nickase activities of the CRISPR-Cas nucleases Cpfl and Cas9. Nat Microbiol. 2019; 4(5): 888-897. DOI: https://doi.org/10.1038/s41564-019-0382-0; PMID: 30833733 PMCID: PMC6512873

22. Shalem O, Sanjana NE, Hartenian E, Shi X, Scott DA, et al. Genome-scale CRISPR-Cas9 knockout screening in human cells. Science. 2014; 343(6166): 84-87. DOI: https://doi.org/10.1126/science.1247005; PMID: 24336571; PMCID: PMC4089965

23. Rezaei H, Farahani N, Hosseingholi EZ, Sathyapalan $\mathrm{T}$ and Hossein Sahebkar A. Harnessing CRISPR/Cas9 technology in cardiovascular disease. Trends Cardiovasc Med. 2019; 30(2): 93-101. PMID: 30935726; DOI: https://doi.org/10.1016/j.tcm.2019.03.005

24. Wang W, Zhang L, Wang X and Zeng Y. The advances in CRISPR technology and 3D genome. Semin Cell Dev Biol. 2019 Jun; 90:54-61. Epub 2018 Aug 25. PMID: 30004018; DOI: https://doi.org/10.1016/j.semcdb.2018.07.009.

25. Ai J-W, Zhou $\mathrm{X}, \mathrm{Xu} \mathrm{T}$, Yang M, Chen $\mathrm{Y}$, et al. CRISPR-based rapid and ultra-sensitive diagnostic test for Mycobacterium tuberculosis. Emerg Microbes Infect. 2019; 8(1): 1361-1369. PMID: 31522608; PMCID: PMC6758691; DOI: https://doi.org/10.1080/22221751.2019.1664939

26. Strich JR and Chertow DS. CRISPR-Cas biology and its application to infectious diseases. Diseases. J Clin Microbiol. 2019; 57(4): e01307-18. PMID: 30429256; PMCID: PMC6440769; DOI: https://doi.org/doi.org/10.1128/JCM.01307-18

27. Hui DS and Zumla A. Severe Acute Respiratory Syndrome: Historical, Epidemiologic, and Clinical Features. Infect Dis Clin North Am. 2019; 33(4): 869-889. DOI: https://doi.org/10.1016/j.idc.2019.07.001

28. Azhar EI, Hui DS, Memish ZA, Drosten C and Zumla A. The Middle East Respiratory Syndrome (MERS). Infect Dis Clin North Am. 2019; 33(4): 891-905. DOI: https://doi.org/10.18683/germs.2019.1155; PMID: 31668197; PMCID: PMC7127753

29. Organization WH. Novel Coronavirus (2019-nCoV): situation $\quad$ report, 3.2020. https://www.who.int/emergencies/diseases/novel-coronavirus-2019/situation-reports

30. Huang C, Wang Y, Li X, Ren L, Zhao J, et al. Clinical features of patients infected with 2019 novel coronavirus in Wuhan, China. Lancet. 2020; 395(10223): 497-506. DOI: https://doi.org/10.1016/S0140-6736(20)30183-5; PMID: 31986264 PMCID: PMC7159299

31. Zumla A, Hui DS, Azhar EI, Memish ZA and Maeurer M. Reducing mortality from 2019-nCoV: host-directed therapies should be an option. Lancet. 2020; 395(10224): e35-e36. PMID: 32035018; PMCID: PMC7133595; DOI: https://doi.org/10.1016/So1406736(20)30305-6

32. Li G, Fan Y, Lai Y, Han T, Li Z, et al. Coronavirus infections and immune responses. J Med Virol. 2020; 92(4):424-432. PMID: 31981224 PMCID: PMC7166547; DOI: https://doi.org/10.1002/jmv.25685

33. Channappanavar R and Perlman S. Pathogenic human coronavirus infections: causes and consequences of cytokine storm and immunopathology. Semin Immunopathol. 2017. 39(5): 529-539. PMID: 28466096; PMCID: PMC7079893; DOI: https://doi.org/10.1007/s00281-017-0629-X

34. Batawi S, Tarazan N, Al-Raddadi R, Al Qasim E, Sindi A, et al. Quality of life reported by survivors after hospitalization for Middle East respiratory syndrome (MERS). Health Qual Life Outcomes. 2019; 17(1):101. PMID: 31186042; PMCID: PMC6560892; DOI: https://doi.org/10.1186/s12955-019-1165-2

35. Ngai JC, Ko FW, Ng SS, TO KW, Tong M, et al. The long-term impact of severe acute respiratory syndrome on pulmonary function, exercise capacity and health status. Respirology. 2010; 15(3): 543-550. PMID: 20337995; PMCID: PMC7192220; DOI: https://doi.org/10.1111/j.1440-1843.2010.01720.x

36. Zumla A, Chan JF, Azhar EI, Hui DS and Yuen K-Y. Coronaviruses-drug discovery and therapeutic options. Nat Rev Drug Discov. 2016; 15(5):327-47. PMID: 26868298; PMCID: PMC7097181; DOI: https://doi.org/10.1038/nrd.2015.37

37. Beigel JH, Nam HH, Adams PL, Krafft A, Ince WL, et al. Advances in respiratory virus therapeutics-A meeting report from the 6th isirv Antiviral Group conference. Antiviral Res. 2019 Jul; 167:45-67. PMID: 30974127; PMCID: PMC7132446; DOI: https://doi.org/10.1016/j.antiviral.2019.04.006

38. Zumla A, Azhar EI, Arabi Y, Alotaibi B, Rao M, et al. Host-directed therapies for improving poor treatment outcomes associated with the middle east respiratory syndrome coronavirus infections. Int J Infect Dis. 2015 Nov;40:71-4. PMID: 26365771; PMCID: PMC7128983; DOI: https://doi.org/10.1016/j.ijid.2015.09.005.

39. Gorbalenya AE, Enjuanes L, Ziebuhr J and Snijder EJ. Nidovirales: Evolving the largest RNA virus genome. Virus Res. 2006; 117(1):17-37. PMID: 16503362; PMCID: PMC7114179; DOI: https://doi.org/10.1016/j.virusres.2006.01.017

40. Nga PT, del Carmen Parquet $M$, Lauber C, Parida M, Nabeshima $T$, et al. Discovery of the first insect nidovirus, a missing evolutionary link in the emergence of the largest RNA virus genomes. PLoS Pathog. 2011; 7(9):e1002215. PMID: 21931546; PMCID: PMC3169540. DOI: https://doi.org/10.1371/journal.ppat.1002215

41. de Wit E, van Doremalen N, Falzarano D and Munster VJ. SARS and MERS: recent insights into emerging coronaviruses. Nat Rev Microbiol. 2016; 14(8):523-34. PMID: 27344959; PMCID: PMC7097822. DOI: https://doi.org/10.1038/nrmicro.2016.81

42. Snijder EJ, Kikkert MandFang Y. Arterivirus molecular biology and pathogenesis. J Gen Virol. 2013; 94(Pt 10):2141-2163. PMID: 23939974; DOI: https://doi.org/10.1099/vir.0.056341-0

43. Gosert R, Kanjanahaluethai A, Egger D, Bienz K and Baker SC. RNA replication of mouse hepatitis virus takes place at doublemembrane vesicles. J Virol. 2002; 76(8):3697-708. PMID: 11907209; PMCID: PMC136101. DOI: https://doi.org/10.1128/JVI.76.8.3697-3708.2002

44. Hagemeijer MC, Vonk AM, Monastyrska I, Rottier PJ and de Haan CA. Visualizing coronavirus RNA synthesis in time by using click chemistry. J Virol. 2012; 86(10):5808-16. PMID: 22438542; PMCID: PMC3347275; DOI: https://doi.org/10.1128/JVI.07207-11 
45. Pedersen KW, van der Meer Y, Roos N, Snijder EJ. Open reading frame la-encoded subunits of the arterivirus replicase induce endoplasmic reticulum-derived double-membrane vesicles which carry the viral replication complex. J Virol. 1999; 73(3):201626. DOI: https://doi.org/10.1128/JVI.73.3.2016-2026.1999; PMID: 9971782; PMCID: PMC104444

46. van Hemert $\mathrm{MJ}$, van den Worm SHE, Knoops $\mathrm{K}$, Mommaas AM, Gorbalenya AE, et al. SARS-Coronavirus Replication/Transcription Complexes Are Membrane-Protected and Need a Host Factor for Activity In Vitro. PLOS Pathog, 2008; 4(5): e1000054. PMID: 18451981; PMCID: PMC2322833; DOI: https://doi.org/10.1371/journal.ppat.1000054

47. Knoops K, Bárcena M, Limpens RW, Koster AJ, Mommaas AM, et al. Ultrastructural characterization of arterivirus replication structures: reshaping the endoplasmic reticulum to accommodate viral RNA synthesis. J Virol. 2012; 86(5):2474-87. PMID: 22190716; PMCID: PMC3302280; DOI: https://doi.org/10.1128/JVI.06677-11

48. Maier HJ, Hawes PC, Cottam EM, Mantell J, Verkade P, et al. Infectious bronchitis virus generates spherules from zippered endoplasmic reticulum membranes. mBio. 2013 Oct 22;4(5):e00801-13. PMID: 24149513; PMCID: PMC3812713; DOI: https://doi.org/10.1128/mBio.00801-13

49. Ulasli $\mathrm{M}$, Verheije $\mathrm{MH}$, de Haan CAM and Reggiori F. Qualitative and quantitative ultrastructural analysis of the membrane rearrangements induced by coronavirus. Cell Microbiol. 2010; 12(6):844-61. PMID: 20088951; PMCID: PMC7159092; DOI: $10.1111 / j .1462-5822.2010 .01437 . x$

50. de Wilde AH, Snijder EJ, Kikkert Mandvan Hemert MJ. Host factors in coronavirus replication. Curr Top Microbiol Immunol. 2018;419:1-42. PMID: 28643204; PMCID: PMC7119980; DOI: https://doi.org/10.1007/82_2017_25

51. van der Hoeven B, Oudshoorn D, Koster AJ, Snijder EJ, Kikkert M, et al. Biogenesis and architecture of arterivirus replication organelles. Virus Res. 2016; 220:70-90. PMID: 27071852; PMCID: PMC7111217; DOI: https://doi.org/10.1016/j.virusres.2016.04.001

52. Zhong Y, Tan YW and Liu DX. Recent Progress in Studies of Arterivirus- and Coronavirus-Host Interactions. Viruses. 2012 Jun;4(6):980-1010. PMID: 22816036; PMCID: PMC3397358; DOI: https://doi.org/10.3390/v4060980

53. Naoumov NV. Cyclophilin inhibition as potential therapy for liver diseases. J Hepatol. 2014 Nov;61(5):1166-74. Epub 2014 Jul 15. PMID: 25048953; DOI: https://doi.org/10.1016/j.jhep.2014.07.008

54. Nigro P, Pompilio G and Capogrossi MC. Cyclophilin A: a key player for human disease. Cell Death Dis. 2013 Oct 31;4(10):e888. PMID: 24176846; PMCID: PMC3920964; DOI: https://doi.org/10.1038/cddis.2013.410

55. Hopkins S and Gallay PA. The role of immunophilins in viral infection. Biochim Biophys Acta. 2015 Oct;1850(10):2103-10. Epub 2014 Nov 18. PMID: 25445708; PMCID: PMC4491039; DOI: https://doi.org/10.1016/j.bbagen.2014.11.011

56. Carbajo-Lozoya J, Ma-Lauer Y, Malešević M, Theuerkorn M, Kahlert V, et al. Human coronavirus NL63 replication is cyclophilin A-dependent and inhibited by non-immunosuppressive cyclosporine A-derivatives including Alisporivir. Virus Res. 2014; 184:44-53. Epub 2014 Feb 22. PMID: 24566223; PMCID: PMC7114444; DOI: https://doi.org/10.1016/j.virusres.2014.02.010

57. de Wilde AH, Falzarano D, Zevenhoven-Dobbe JC, Beugeling C, Fett C, et al. Alisporivir inhibits MERS- and SARS-coronavirus replication in cell culture, but not SARS-coronavirus infection in a mouse model. Virus Res. 2017; 228:7-13. Epub 2016 Nov 10. PMID: 27840112; PMCID: PMC7114565; DOI: https://doi.org/10.1016/j.virusres.2016.11.011

58. de Wilde AH, Raj VS, Oudshoorn D, Bestebroer TM, van Nieuwkoop S, et al. MERS-coronavirus replication induces severe in vitro cytopathology and is strongly inhibited by cyclosporin A or interferon- $\alpha$ treatment. J Gen Virol. 2013; 94(Pt 8):1749-1760. Epub 2013 Apr 25. PMID: 23620378; PMCID: PMC3749523; DOI: https://doi.org/10.1099/vir.0.052910-0

59. de Wilde AH, Zevenhoven-Dobbe JC, van der Meer Y, Thiel V, Narayanan K, et al. Cyclosporin A inhibits the replication of diverse coronaviruses. J Gen Virol. 2011; 92(Pt 11):2542-2548. Epub 2011 Jul 13. PMID: 21752960; PMCID: PMC3352363; DOI: https://doi.org/10.1099/vir.0.034983-0

6o. Kim Y, Lee C. Porcine epidemic diarrhea virus induces caspase-independent apoptosis through activation of mitochondrial apoptosis-inducing factor. Virology. 2014; 460-461:180-93. Epub 2014 Jun 5. PMID: 25010284; PMCID: PMC7127720. DOI: https://doi.org/10.1016/j.virol.2014.04.040

61. Tanaka Y, Sato Y, Osawa S, Inoue M, Tanaka S, et al. Suppression of feline coronavirus replication in vitro by cyclosporin A. Vet Res. 2012 Apr 30; 43(1):41. PMID: 22546085; PMCID: PMC3403912; DOI: https://doi.org/10.1186/1297-9716-43-41

62. von Brunn A. Editorial overview: Engineering for viral resistance. Current Opinion in Virology، 2015. 14. von Brunn A. Editorial overview: Engineering for viral resistance. Curr Opin Virol. 2015; 14:v-vii. Epub 2015 Aug 28. PMID: 26320757; PMCID: PMC7128793; DOI: https://doi.org/10.1016/j.coviro.2015.08.009

63. von Brunn A, Ciesek S, von Brunn B and Carbajo-Lozoya J. Genetic deficiency and polymorphisms of cyclophilin A reveal its essential role for Human Coronavirus 229E replication. Curr Opin Virol. 2015 Oct;14:56-61. Epub 2015 Aug 27. PMID: 26318518; PMCID: PMC7102849; DOI: https://doi.org/10.1016/j.coviro.2015.08.004

64. Tanaka Y, Sato Y and Sasaki T. Feline coronavirus replication is affected by both cyclophilin A and cyclophilin B. J Gen Virol. 2017 Feb; 98(2):190-200. Epub 2017 Mar 13. PMID: 27902373; PMCID: PMC7079567. DOI: https://doi.org/10.1099/jgv.0.000663

65. de Wilde $A H$, Li Y, van der Meer Y, Vuagniaux G, Lysek R, et al. Cyclophilin inhibitors block arterivirus replication by interfering with viral RNA synthesis. J Virol. 2013 Feb; 87(3):1454-64. Epub 2012 Nov 14. PMID: 23152531; PMCID: PMC3554155. DOI: https://doi.org/10.1128/JVI.02078-12

66. Foss DV, Hochstrasser ML and Wilson RC. Clinical applications of CRISPR-based genome editing and diagnostics. Transfusion. 2019; 59(4):1389-1399. Epub 2019 Jan 2. PMID: 30600536; DOI: https://doi.org/10.1111/trf.15126

67. Brokowski C and Adli M. CRISPR ethics: moral considerations for applications of a powerful tool. J Mol Biol. 2019 Jan 4; 431(1):88-101. Epub 2018 Jun 7. PMID: 29885329; PMCID: PMC6286228; DOI: https://doi.org/10.1016/j.jmb.2018.05.044

68. Sugarman J. Ethics and germline gene editing. EMBO Rep. 2015 Aug;16(8):879-80. Epub 2015 Jul 2. PMID: 26138102; PMCID: PMC4552475; DOI: https://doi.org/10.15252/embr.201540879

69. Brokowski C. Do CRISPR germline ethics statements cut it? CRISPR J. 2018; 1(2):115-125. PMID: 31021208; PMCID: PMC6694771; DOI: https://doi.org/10.1089/crispr.2017.0024 